\title{
On the Analytic Structure of the Quark Self-Energy in Nambu-Jona-Lasinio Models
}

\author{
P. P. Domitrovich and H. Müther \\ Institut für Theoretische Physik, \\ Universität Tübingen, \\ D-72076 Tübingen, Germany
}

September 22, 2018

September 22, 2018

\begin{abstract}
The self-energy of quarks is investigated for various models which are inspired by the Nambu-Jona-Lasinio (NJL) model. Including, beyond the HartreeFock approximation, terms up to second-order in the quark interaction, the real and imaginary parts of scalar and vector components of the self-energy are discussed. The second-order contributions depend on the energy and momentum of the quark under consideration. This leads to solutions of the Dirac equation which are significantly different from those of a free quark or a quark with constant effective mass, as obtained in the Hartree-Fock approximation.
\end{abstract}




\section{Introduction}

For low momentum transfer between quarks, where asymptotic freedom of QCD is no longer applicable, the Nambu-Jona-Lasinio (NJL) model [1], reinterpreted for quarks, has received much attention during the last few years [2]. This model utilizes an effective two-body quark-quark interaction and concomitantly displays several of the essential features of QCD. For example, the interaction part of the NJL Lagrangian exhibits chiral symmetry, and for interaction strengths beyond a critical value this symmetry is broken dynamically. The breaking of chiral symmetry, which is obtained in the mean-field approximation, yields a sizable quark-antiquark pair condensation and leads to constituent quark masses which are much larger than the bare current quark masses considered in the Lagrangian. Although confinement is not included in the NJL model, this model has been successful in accounting for important features of QCD at low energies and can be considered as a low-energy approximation to QCD [3].

In most cases, the NJL model is treated in the mean-field or Hartree-Fock approximation in order to study quark properties. As already mentioned above, a large scalar contribution to the self-energy of the quarks results and gives rise to an effective mass. It is easy to adjust the parameters of the model, strength of the interaction, and cutoff parameter in such a way that the effective masses are close to the empirical masses of the constituent quarks [4, 5, 6. As a next step one can study the properties of the mesons as predicted by this model. For that purpose one uses the single-quark Green's function, as determined in the Hartree-Fock approximation, and evaluates the polarization propagator or response function for the various quark - antiquark $(q \bar{q})$ channels within the RPA approximation. The residual interaction yields very collective excitation modes, in particular, for the isovector-pseudoscalar and the scalar-isoscalar excitations, and the energies of these collective modes are associated with the masses of the corresponding mesons ( $\pi$ and $\sigma$ mesons).

As the quark interaction of the NJL model gives rise to such collective $q \bar{q}$ excitation modes, it seems quite natural to go beyond the mean-field approximation and study the effects of the interaction of quarks with the $q \bar{q}$ excitations on the self-energy of quarks. One approach in this direction is to consider the effects arising from the $\pi$ exchange term [0]. Another way is to evaluate all contributions of second order in the interaction (see Figure 1) using standard many-body theory techniques [9, 10]. These two approaches supplement each other in the sense that the former one only accounts for one special collective $q \bar{q}$ mode, whereas the second approach considers all $q \bar{q}$ excitations, ignoring the collective features which are due to the residual interaction.

The effects of the second-order terms on the real, scalar part of the self-energy have been investigated in a previous paper [8]. These contributions depend on the energy and momentum of the quark under consideration, and it was found that, depending on the details of the Lagrangian under consideration, the effects can be a sizable correction of the Hartree-Fock contribution. In contrast to the Hartree-Fock 
approximation, however, the second-order terms also yield a vector component for the self-energy and imaginary parts for both the scalar and vector components of the self-energy.

The terms displayed in Figure 1 describe the admixture of $2 q-1 \bar{q}$ and $2 \bar{q}-1 q$ components to the propagation of the quarks and antiquarks, respectively. Therefore, after one has determined the complete analytic structure of the quark self-energy on this level, one can determine the corresponding Green's function and evaluate the $q \bar{q}$ response function. In this way one accounts for the admixture of $2 q-2 \bar{q}$ configurations to the polarization propagator, which should provide a more realistic description, in particular, for the mesons like the $\sigma$, which contain large $2 \pi$ contributions. Therefore, the present investigation can be understood as a step towards a consistent treatment of the NJL model beyond the mean-field approximation (see also discussion in [8]).

As in our earlier paper [8], four Lagrangians will be considered, adjusting the coupling strength in such a way that the same constituent mass is obtained for quarks of zero momentum in each model. In Section 2, a brief description of the four Lagrangians is presented, while in Section 3, the formulas for the scalar and vector self-energies are discussed. Section 4 contains the results of the calculations accompanied by a discussion of these results, and the main conclusions are summarized in Section 5 .

\section{Lagrangians motivated by the NJL model}

In its original form [1], the NJL model was designed to describe a system of interacting nucleons, but has more recently been reinterpreted as a quark Lagrangian of identical form:

$$
\mathcal{L}_{D}^{(A)}=\bar{\psi}\left(i \not \nabla-m_{0}\right) \psi+G_{A}\left[(\bar{\psi} \psi)^{2}+\left(\bar{\psi} i \gamma^{5} \vec{\tau} \psi\right)^{2}\right],
$$

such that the Fermion field $\psi$ now represents quarks with $\mathrm{SU}(2)$ flavor $(\vec{\tau}$ denoting the Pauli matrices for the flavor degrees of freedom) and SU(3) color. For vanishing current quark mass $m_{0}$, this Lagrangian is invariant under a chiral transformation. Particular to this study, A denotes the first of the four models considered and the subscript $D$ implies that only the direct part of the contact interaction defined in eq.(11) is included.

For such a contact interaction, the Pauli-exchange terms originating from the Lagrangian in eq.(11) are easily obtained by performing a Fierz transformation (in Dirac, flavor, and color spaces) of the interaction term

$$
G_{A}\left[(\bar{\psi} \psi)^{2}+\left(\bar{\psi} i \gamma^{5} \vec{\tau} \psi\right)^{2}\right] \stackrel{\text { Fierz }}{\longrightarrow} \mathcal{L}_{E}^{(A)},
$$

and defining a total Lagrangian

$$
\mathcal{L}^{(A)}=\mathcal{L}_{D}^{(A)}-\mathcal{L}_{E}^{(A)}
$$


The exchange terms of the original Lagrangian defined in eq.(位) are now included by evaluating the contributions of all terms in eq.(3). The second-order mass contribution for this Lagrangian is small [8] and dominated by the second-order self-energy for the pseudovector interaction, while the second-order self-energy contributions for the scalar and vector interactions essentially cancel.

Model B is defined by the Lagrangian:

$$
\mathcal{L}_{D}^{(B)}=\bar{\psi}\left(i \not \nabla-m_{0}\right) \psi-G_{B}\left(\bar{\psi} \gamma_{\mu} \psi\right)^{2} .
$$

The interaction term defined in this Lagrangian is symmetric under a chiral transformation and is a scalar in color space. The exchange terms are determined in a way analogous to eq.(3). This Lagrangian yields the largest second-order effect in which the real part of the scalar self-energy is dominated by the coupling to vector $q \bar{q}$ excitations.

The Lagrangian of model C:

$$
\mathcal{L}_{D}^{(C)}=\bar{\psi}\left(i \not \nabla-m_{0}\right) \psi-G_{C}\left(\bar{\psi} \gamma_{\mu} \vec{\lambda} \psi\right)^{2},
$$

may be of special interest as it can be motivated by QCD. Starting from the path integral formulation of QCD, attempts have been made to eliminate the gluon degrees of freedom by trying to derive or motivate effective Lagrangians for quarks in the low-energy domain. Employing a current expansion of the effective quark action [11], or using the field strength approach [12, 13], one obtains a quark-quark interaction defined in terms of color-vector currents. Using the nomenclature introduced before, the corresponding NJL type Lagrangian exhibits the form of eq.(5). Here the second-order mass contribution is also dominated by the vector interaction for this Lagrangian.

Finally, Lagrangian D provides second-order mass contributions similar to that of Lagrangian A, but significantly larger. This Lagrangian has also been considered in ref. [14], but with a momentum dependent coupling constant:

$$
\mathcal{L}_{D}^{(D)}=\bar{\psi}\left(i \not \nabla-m_{0}\right) \psi+G_{D}\left[(\bar{\psi} \vec{\lambda} \psi)^{2}+\left(\bar{\psi} i \gamma^{5} \vec{\tau} \vec{\lambda} \psi\right)^{2}\right] .
$$

Each of the Lagrangians considered are nonrenormalizable, due to the contact interaction. Therefore one has to introduce a cutoff scheme to regularize the various contributions. In this work a three-momentum noncovariant cutoff scheme is utilized. Hence, for each directed line in the second-order self-energy (Fig. 1), which represents a single-particle propagator, the appropriate three-momenta squared are restricted to values less than the cutoff parameter $\Lambda$ squared, while the zeroth components of the momenta are unrestricted.

Applying the same cutoff procedure to the evaluation of the Hartree-Fock contribution to the self-energy, one obtains a non-linear gap equation to determine the effective quark mass in this approximation:

$$
m_{H F}^{*}=m_{0}+\tilde{G} \frac{24}{(2 \pi)^{3}} \int_{0}^{\Lambda} d^{3} p \frac{m_{H F}^{*}}{\sqrt{\mathbf{p}^{2}+m_{H F}^{*}}} .
$$


The coupling constant $\tilde{G}$ refers to the coupling constant in front of the scalar - isoscalar - colorscalar term of the Lagrangian including the effects of the exchange term in eq.(33). For the various models we obtain:

$$
\tilde{G}=\frac{13}{12} G_{A}=\frac{1}{6} G_{B}=\frac{8}{9} G_{C}=\frac{4}{9} G_{D} .
$$

Hence, different strengths for the residual interaction in the various channels result when the coupling constants are adjusted so that the Hartree-Fock approximation leads to the very same gap equation for each model. As in our previous paper [8] the coupling constants $G_{i}$ for the various models $i=A, B, C, D$ are adjusted in such a way that the effective mass calculated from the Hartree-Fock plus the second order terms is $313 \mathrm{MeV}$ for a quark of momentum $|\vec{p}|=0$. The cutoff parameter $\Lambda=653$ $\mathrm{MeV}$ has been chosen for all models.

\section{Calculation of the Self-Energy}

The second-order contributions to the self-energy of the quarks can be split into various parts $\Sigma^{(\lambda)}$, where the index $\lambda$ refers to the quantum numbers of the $q \bar{q}$ excitation considered. We distinguish $\lambda=s$ for scalar, $\lambda=p s$ for pseudoscalar, and $\lambda=v$ for vector excitations. The distinction of the $q \bar{q}$ excitations with respect to the flavor and color quantum numbers will be discussed at the end of this section.

For a given excitation mode, $\lambda$, the scalar contribution of the second-order selfenergy is obtained as follows:

$$
\delta \Sigma_{s}^{(\lambda)}=\frac{1}{4 n_{f} n_{c}} \operatorname{tr}\left[\delta \Sigma^{(\lambda)}\right]
$$

where $n_{f}$ and $n_{c}$ are the number of flavors and colors, respectively. All traces in this paper are over spin (Dirac), color, and flavor spaces.

In [8] it has been shown that the real part of these self-energy contributions, $\delta \Sigma_{s}^{(\lambda)}$, can be evaluated from irreducible response functions $\Pi_{\lambda}^{(0)}$ of the corresponding excitation mode. The real and imaginary parts of $\Pi_{\lambda}^{(0)}$ are related by a dispersion relation:

$$
\operatorname{Re} \Pi_{\lambda}^{(0)}(q)=\frac{1}{\pi} \mathrm{P} \int_{0}^{\infty} d q_{0}^{\prime} \operatorname{Im} \Pi_{\lambda}^{(0)}\left(q_{0}^{\prime}, \mathbf{q}\right)\left[\frac{1}{q_{0}^{\prime}-q_{0}}+\frac{1}{q_{0}^{\prime}+q_{0}}\right],
$$

and also the real and imaginary parts are even functions of the energy variable $q_{0}$. Therefore the second-order contributions to the scalar self-energy can be determined by knowing only the imaginary part of the polarization propagator. As an example, we recall the result for $\delta \Sigma_{s}^{(s)}$, the scalar contribution to the self-energy originating from a pure scalar interaction in a Lagrangian of the form:

$$
\delta \mathcal{L}=\tilde{G}(\bar{\psi} \psi)^{2} .
$$


Approximating the Green's functions needed for the evaluation of the second-order diagram by those of a quark with a constant mass $m^{*}$, one obtains:

$$
\begin{aligned}
& \delta \Sigma_{s}^{(s)}(p)=-\frac{\tilde{G} m^{*}}{4 \pi^{3}} \int_{0}^{\Lambda} \frac{\mathbf{q}^{2} d q}{E_{q}^{*}} \int_{-1}^{+1} d x \\
& \quad \times\left\{\int_{0}^{\infty} d q_{0} \frac{\operatorname{Im} \Pi_{s}^{(0)}\left(q_{0},|\mathbf{p}-\mathbf{q}|\right)}{q_{0}+p_{0}+E_{q}^{*}+i \epsilon}+\int_{0}^{\infty} d q_{0} \frac{\operatorname{Im} \Pi_{s}^{(0)}\left(q_{0},|\mathbf{p}-\mathbf{q}|\right)}{q_{0}-p_{0}+E_{q}^{*}-i \epsilon}\right\} .
\end{aligned}
$$

The nomenclature used in this equation corresponds to the assignment of momenta in Fig. 1. The integration variable $x$ represents the cosine of the angle between $\mathbf{p}$ and q. The imaginary part of the scalar response function is:

$$
\begin{aligned}
\operatorname{Im}_{s}^{(0)}(q)= & \frac{\tilde{G} n_{f} n_{c}}{4 \pi^{2}} \int \frac{d^{3} k}{E_{k}^{*} E_{k-q}^{*}}\left(E_{k}^{*} E_{k-q}^{*}+\mathbf{k}(\mathbf{k}-\mathbf{q})-m^{* 2}\right) \\
& \times \delta\left(\left|q_{0}\right|-E_{k}^{*}-E_{k-q}^{*}\right) \Theta(\Lambda-|\mathbf{k}|) \Theta(\Lambda-|\mathbf{k}-\mathbf{q}|)
\end{aligned}
$$

with

$$
E_{k}^{*}=\sqrt{\mathbf{k}^{2}+m^{* 2}}
$$

This self-energy result is a simple extension of the real part found previously [8]. Eq.(12) furthermore demonstrates that the imaginary part is:

$$
\begin{aligned}
\delta \operatorname{Im} \Sigma_{s}^{(s)}(p)= & -\operatorname{sgn}\left(p_{0}\right) \Theta\left(p_{0}^{2}-m^{* 2}\right) \frac{\tilde{G} m^{*}}{4 \pi^{2}} \\
& \times \int_{0}^{\min \left(\Lambda, \sqrt{p_{0}^{2}-m^{* 2}}\right)} \frac{\mathbf{q}^{2} d q}{E_{q}^{*}} \int_{-1}^{+1} d x \operatorname{Im} \Pi_{s}^{(0)}\left(\left|p_{0}\right|-E_{q}^{*},|\mathbf{p}-\mathbf{q}|\right),
\end{aligned}
$$

and also that the real and imaginary parts of the scalar contribution to the self-energy are related by a dispersion relation:

$$
\delta \operatorname{Re} \Sigma_{s}^{(s)}(q)=\frac{1}{\pi} \mathrm{P} \int_{0}^{\infty} d q_{0}^{\prime} \delta \operatorname{Im} \Sigma_{s}^{(s)}\left(q_{0}^{\prime}, \mathbf{q}\right)\left[\frac{1}{q_{0}^{\prime}-q_{0}}+\frac{1}{q_{0}^{\prime}+q_{0}}\right] .
$$

Hence, we find that the imaginary part $\delta \operatorname{Im} \Sigma_{s}^{(s)}(p)$ yields an odd function of $p_{0}$ for a given $|\mathbf{p}|$ (see eq.(15)), while the real part is clearly even (see eq.(16)). As the expressions obtained for the scalar contributions originating from pseudoscalar $(\lambda=$ ps) and vector excitation modes $(\lambda=v)$ are similar to eq.(12) (see [8]), the dispersion relation of eq.(16) is also valid for these modes. One finds in general for all excitation modes $\lambda$ that, for a given $|\mathbf{p}|$, the real part of $\delta \Sigma_{s}^{(\lambda)}(p)$ is identical for $p_{0}$ and $-p_{0}$, while the imaginary part changes sign. 
We now turn to the time-like vector contributions to the second-order self-energy originating from the various excitation modes $\lambda$. These contributions are defined by:

$$
\delta \Sigma_{0}^{(\lambda)}=\frac{-1}{4 n_{f} n_{c}} \operatorname{tr}\left[\gamma^{0} \delta \Sigma^{(\lambda)}\right]
$$

(compare eq.(9)). Note that the Hartree-Fock approximation does not provide a vector contribution to the self-energy for quarks in the vacuum. Each complex selfenergy originating from the second-order terms is derived in a manner similar to that used to find the real part of the scalar self-energies [8].

In the case of scalar excitation modes $(\lambda=s)$, assuming a scalar interaction as described by the Lagrangian in eq.(11), the result is:

$$
\begin{aligned}
\delta \Sigma_{0}^{(s)}(p)= & \frac{i \tilde{G}}{2} \int \frac{d^{4} q}{(2 \pi)^{4}} \operatorname{tr}\left[\gamma^{0} g(q)\right] \Pi_{s}^{(0)}(p-q) \\
= & \frac{-\tilde{G}}{4 \pi^{3}} \int_{0}^{\Lambda} \mathbf{q}^{2} d q \int_{-1}^{+1} d x \\
& \times\left\{\int_{0}^{\infty} d q_{0} \frac{\operatorname{Im} \Pi_{s}^{(0)}\left(q_{0},|\mathbf{p}-\mathbf{q}|\right)}{q_{0}+p_{0}+E_{q}^{*}+i \epsilon}-\int_{0}^{\infty} d q_{0} \frac{\operatorname{Im} \Pi_{s}^{(0)}\left(q_{0},|\mathbf{p}-\mathbf{q}|\right)}{q_{0}-p_{0}+E_{q}^{*}-i \epsilon}\right\} .
\end{aligned}
$$

This result is quite similar to the corresponding result for the scalar self-energy (see eq.(12)) except for the facts that a factor $m^{*} / E_{q}^{*}$ is missing and a minus sign occurs in front of the second principal value integral. Taking the real part of eq.(19), an odd function of $p_{0}$ for fixed $|\mathbf{p}|$ is obtained. The imaginary part of eq.(19) is:

$$
\begin{aligned}
\delta \operatorname{Im} \Sigma_{0}^{(s)}(p)= & \Theta\left(p_{0}^{2}-m^{* 2}\right) \frac{\tilde{G}}{4 \pi^{2}} \\
& \times \int_{0}^{\min \left(\Lambda, \sqrt{p_{0}^{2}-m^{* 2}}\right)} \mathbf{q}^{2} d q \int_{-1}^{+1} d x \operatorname{Im} \Pi_{s}^{(0)}\left(\left|p_{0}\right|-E_{q}^{*},|\mathbf{p}-\mathbf{q}|\right) .
\end{aligned}
$$

The imaginary part clearly is an even function.

The vector second-order self-energy for a purely pseudoscalar interaction is:

$$
\delta \Sigma_{0}^{(p s)}(p)=\frac{-i \tilde{G}}{2} \int \frac{d^{4} q}{(2 \pi)^{4}} \operatorname{tr}\left[\gamma^{0} g(q)\right] \Pi_{p s}^{(0)}(p-q) .
$$

Comparing eq.(18) and eq.(21), the complex self-energy and its imaginary part in this case can be found by using eq.(19) and eq.(20), respectively. For the vector self-energy with a pure scalar interaction, a positive value is obtained for energies less than the cutoff, and the vector self-energy with a pseudoscalar interaction provides a negative result in the same energy domain. 
The vector self-energy for the case of a pure vector interaction is more easily calculated by using different momenta than those shown in Fig. 1. For this case, $p$ and $p-q$ are simply exchanged. Then,

$$
\begin{aligned}
\delta \Sigma_{0}^{(v)}(p)= & \frac{-i \tilde{G}}{4} \int \frac{d^{4} q}{(2 \pi)^{4}} \sum_{\theta, \phi} \operatorname{tr}\left[\gamma^{0} \gamma_{\theta} g(p-q) \gamma^{\phi}\right] \Pi_{v, \phi}^{(0) \theta}(q) \\
= & \frac{\tilde{G}}{4 \pi^{3}} \int_{-1}^{+1} d x \int_{0}^{|\mathbf{p}| x+\sqrt{\Lambda^{2}-|\mathbf{p}|^{2}\left(1-x^{2}\right)}} \mathbf{q}^{2} d q \\
& \times\left\{\int_{0}^{\infty} d q_{0} \frac{\operatorname{Im~f} f_{v}^{(0)}\left(q_{0},|\mathbf{p}|,|\mathbf{q}|, x\right)}{q_{0}+p_{0}+E_{p-q}^{*}+i \epsilon}-\int_{0}^{\infty} d q_{0} \frac{\operatorname{Imf}_{v}^{(0)}\left(q_{0},|\mathbf{p}|,|\mathbf{q}|, x\right)}{q_{0}-p_{0}+E_{p-q}^{*}-i \epsilon}\right\}
\end{aligned}
$$

for $|\mathbf{p}|<\Lambda$. For the case of $|\mathbf{p}| \geq \Lambda$, the lower limit of the integral over $x$ is replaced by $\sqrt{1-\left(\frac{\Lambda}{|\mathbf{p}|}\right)^{2}}$. The imaginary part of this self-energy is:

$$
\delta \operatorname{Im} \Sigma_{0}^{(v)}(p)=-\Theta\left(p_{0}^{2}-m^{* 2}\right) \frac{\tilde{G}}{4 \pi^{2}} \int_{-1}^{+1} d x \int_{0}^{q_{\max }} \mathbf{q}^{2} d q \operatorname{Im} \mathrm{f}_{v}^{(0)}\left(\left|p_{0}\right|-E_{q}^{*},|\mathbf{p}|,|\mathbf{q}|, x\right),
$$

for $|p|<\min \left(\Lambda^{2}, p_{0}^{2}-m^{* 2}\right)$, and

$$
q_{\text {max }}=|\mathbf{p}| x+\min \left[\sqrt{\Lambda^{2}-|\mathbf{p}|^{2}\left(1-x^{2}\right)}, \sqrt{\left(p_{0}^{2}-m^{* 2}\right)-|\mathbf{p}|^{2}\left(1-x^{2}\right)}\right] .
$$

For $|\mathbf{p}| \geq \min \left(\Lambda^{2}, p_{0}{ }^{2}-m^{* 2}\right)$, the lower limit of the $x$ integral is changed in a manner similar to that done in eq.(23) when $|\mathbf{p}| \geq \Lambda$.

The imaginary part of the function $\mathrm{f}$ in this self-energy is given by:

$$
\begin{aligned}
\operatorname{Imf}_{v}^{(0)}\left(q_{0},|\mathbf{p}|,|\mathbf{q}|, x\right)= & \operatorname{Im} \Pi_{v, 0}^{(0) 0}\left(q_{0},|\mathbf{q}|\right) \\
& -\sum_{i=1}^{3}\left[\operatorname{Im} \Pi_{v, i}^{(0) i}\left(q_{0},|\mathbf{q}|\right)-\frac{2}{E_{p-q}^{*}}\left(\mathrm{p}_{i}-\mathrm{q}_{i}\right) \operatorname{Im} \Pi_{v, i}^{(0) 0}\left(q_{0},|\mathbf{q}|\right)\right],
\end{aligned}
$$

and behaves as the response functions encountered here. The various components of the matrix for the vector response function are obtained from the following formula:

$$
\Pi_{v, \phi}^{(0) \theta}(q)=-2 i \tilde{G} \int \frac{d^{4} k}{(2 \pi)^{4}} \operatorname{tr}\left[\gamma^{\theta} g(k) \gamma_{\phi} g(k-q)\right] .
$$

The terms of the function $\mathrm{f}$ in eq.(26) can be separated and written as:

$$
\begin{aligned}
\operatorname{Im} \Pi_{v, 0}^{(0) 0}\left(q_{0},|\mathbf{q}|\right)- & \sum_{i=1}^{3} \operatorname{Im} \Pi_{v, i}^{(0) i}\left(q_{0},|\mathbf{q}|\right)=-\frac{\tilde{G} n_{f} n_{c}}{2 \pi^{2}} \int \frac{d^{3} k}{E_{k}^{*} E_{k-q}^{*}}\left(2 E_{k}^{*} E_{k-q}^{*}+m^{* 2}\right) \\
& \times \delta\left(\left|q_{0}\right|-E_{k}^{*}-E_{k-q}^{*}\right) \Theta(\Lambda-|\mathbf{k}|) \Theta(\Lambda-|\mathbf{k}-\mathbf{q}|),
\end{aligned}
$$


and

$$
\begin{aligned}
\sum_{i=1}^{3}\left(\mathrm{p}_{i}-\mathrm{q}_{i}\right) \operatorname{Im} \Pi_{v, i}^{(0) 0}\left(q_{0},|\mathbf{q}|\right)= & \operatorname{sgn}\left(q_{0}\right) \frac{\tilde{G} n_{f} n_{c}}{4 \pi^{2}} \int \frac{d^{3} k}{E_{k}^{*} E_{k-q}^{*}} \\
& \times\left[(\mathbf{p}-\mathbf{q}) \mathbf{k}\left(E_{k-q}^{*}-E_{k}^{*}\right)+(\mathbf{p}-\mathbf{q}) \mathbf{q} E_{k}^{*}\right] \\
& \times \delta\left(\left|q_{0}\right|-E_{k}^{*}-E_{k-q}^{*}\right) \Theta(\Lambda-|\mathbf{k}|) \Theta(\Lambda-|\mathbf{k}-\mathbf{q}|) .
\end{aligned}
$$

From eqs.(20) and (24) one can see that, for all excitation modes $\lambda$, the imaginary parts of the vector contributions are even functions of $p_{0}$, while eqs.(19) and (23) demonstrate that the real parts are odd functions of $p_{0}$. The real and imaginary parts of the second-order vector self-energies are related by:

$$
\delta \operatorname{Re} \Sigma_{0}^{(\lambda)}(q)=\frac{1}{\pi} \mathrm{P} \int_{0}^{\infty} d q_{0}^{\prime} \delta \operatorname{Im} \Sigma_{0}^{(\lambda)}\left(q_{0}^{\prime}, \mathbf{q}\right)\left[\frac{1}{q_{0}^{\prime}-q_{0}}-\frac{1}{q_{0}^{\prime}+q_{0}}\right] .
$$

Until now we have only considered the Dirac structure of the excitation modes $\lambda$ and the corresponding interaction terms, assuming a scalar for the isospin and color part. For interaction terms which are isovector rather than isoscalar, one obtains the corresponding second-order contribution to the self-energy with an additional factor of 3. Also, for interaction terms which are products of vector operators in $\mathrm{SU}(3)$ color space, the corresponding contributions to the self-energy should be multiplied by a factor of $32 / 9$.

\section{Results and Discussion}

As a first point in the discussion of our results, we would like to consider several of the basic features of the various contributions to the quark self-energy. For that purpose we intially consider in our discussion highly simplified quark-quark interactions which contain either a pure scalar contribution (see eq.(11)), exciting only scalar $q \bar{q}$ excitations, or similarly pure pseudoscalar or vector contributions, while always ignoring the Fock exchange terms. To allow a comparison between these different types of self-energies, we have chosen the same coupling constant and cutoff parameter for these modes $\left(\tilde{G}=1.618 \times 10^{-6} \mathrm{MeV}^{-2}, \Lambda=653 \mathrm{MeV}\right.$, see [8]). We would like to recall that, in calculating the contributions of second-order, we consider a Green's function for quarks with a constant effective mass $m^{*}$ of $313 \mathrm{MeV}$.

Results for the imaginary parts of the scalar and vector self-energies originating from a pure scalar interaction are displayed in Fig. 2. The various momenta $(|\mathbf{p}|=$ 0, 300, and $600 \mathrm{MeV}$ ) are considered for the quarks. It is clear that the imaginary part can be different from zero only for those positive energies $p_{0}$ ranging between the threshold of $2 q-1 \bar{q}$ excitations $\left(3 m^{*}=939 \mathrm{MeV}\right)$ and the largest energy compatible with the cutoff $\left(3 \sqrt{m^{* 2}+\Lambda^{2}} \approx 2170 \mathrm{MeV}\right)$. As can be deduced from eqs.(15) and 
(20), the imaginary part of the scalar self-energy is negative, while the imaginary part of the vector self-energy is positive. Note that, for negative energies $p_{0}$, the imaginary part of the scalar self-energy changes sign, whereas the imaginary part of vector self-energy depends on $\left|p_{0}\right|$ only (see discussion in Sec. 3). The absolute values of the imaginary parts decrease with an increase of the space-like component $|\mathbf{p}|$ of the quark momentum. This leads to a reduction by almost a factor of 2 , if the momentum is increased from $\left|p_{0}\right|=0$ to $\left|p_{0}\right|=600 \mathrm{MeV}$. A quite similar momentum dependence of the imaginary contributions to the self-energy is also observed if other interactions are considered.

Therefore, for the comparison of contributions arising from the three basic kinds of interactions, displayed in Figure 3, results are presented only for $|\mathbf{p}|=0$. It is worth noting that the contributions originating from scalar and pseudoscalar interactions always have opposite sign. Since the coupling strengths of these two kinds of interactions are connected in Lagrangians with chiral symmetry (see, e.g., A and $\mathrm{D}$, defined in eqs.(1) and (6)), these contributions tend to cancel each other. Large contributions are obtained from vector interactions and corresponding $q \bar{q}$ excitations.

As discussed in Section 3, the real and imaginary parts of the second-order contributions to the self-energy are related by the dispersion relations of eq.(16) and eq.(30) for the scalar and vector terms, respectively. From these dispersion relations one can immediately understand the features of the energy-dependence of the real parts of the self-energies shown in Fig. 4, which are related to the corresponding imaginary parts displayed in Fig. 3. It is obvious from eq.(30) that the real vector part of the self-energy is an odd function of the energy variable $p_{0}$ and consequently vanishes for $p_{0}=0$, while the real scalar part is an even function and therefore can be different from 0 also at $p_{0}=0$. With increasing positive energies, the real parts of both the scalar and vector self-energies become larger in absolute value with increasing $p_{0}$ until one approaches energies around $1500 \mathrm{MeV}$, i.e. , energies close to the maximum of the imaginary parts. At these energies the real parts change sign and finally approach zero.

In discussing the mathematical behavior of the real part, one should keep in mind, however, that the maximum of the absolute value of the imaginary part of the secondorder self-energy is primarily determined by the cutoff procedure for the intermediate $2 q-1 \bar{q}$ excitations (see discussion above). Therefore the change in sign of the real part of the self-energy, which is related to this maximum, should not seriously be considered from a physical point of view. Indeed, as we will see below, the on-shell solution of the Dirac equation for quarks with momenta below the cutoff $(|\mathbf{p}|<\Lambda)$ requires self-energies at energies $p_{0}$ below those for which the real part changes sign.

The statements on the cancellation between contributions arising from scalar and pseudoscalar interactions made above for the imaginary part, of course, also apply to the real parts. From the dispersion relations it is furthermore obvious that the various real parts depend on the space-component $\mathbf{p}$ of the quark momentum in a similar way as the corresponding imaginary parts. 
Having discussed several of the basic features of the various contributions to the self-energy arising from the different interaction terms, we now turn to the 4 effective Lagrangians defined as models A, B, C and D in Section 2. For each of these Lagrangians, we calculate the contributions to the quark self-energy of first and second order in the quark - quark interaction and consider the on-shell solutions of the Dirac equation for quarks with this self-energy. For each momentum $|\mathbf{p}|$, these on-shell solutions must fulfill the following energy relation:

$$
\left(p_{0}+\operatorname{Re} \Sigma_{0}^{(2)}\left(p_{0}, \mathbf{p}\right)\right)^{2}=\mathbf{p}^{2}+\left(m_{0}+\operatorname{Re} \Sigma_{s}^{H F}+\operatorname{Re} \Sigma_{s}^{(2)}\left(p_{0}, \mathbf{p}\right)\right)^{2},
$$

where $\Sigma_{0}^{(2)}$ and $\Sigma_{s}^{(2)}$ refer to the vector and scalar components, respectively, obtained from the second-order terms. For each Lagrangian we assume a bare current quark mass of $m=5 \mathrm{MeV}$ and adjust the coupling constant in such a way that the relation of eq.(31) yields an energy $p_{0}$ of $313 \mathrm{MeV}$ for a quark with momentum $|\mathbf{p}|=0$. In order to get an idea of the importance of the various contributions to the self-energy in the different models, we present in Table 1 these on-shell quantities for quarks with zero momentum $|\mathbf{p}|$.

One finds that the relative importance of the second-order contributions as compared to the Hartree-Fock term is quite different in the different Lagrangians under consideration. The effect of the second-order terms is negligibly small for model A, which corresponds to the original NJL Lagrangian (see eq.(便). This is in accord with the observation made above that effects due to a scalar and a pseudoscalar interaction, which are the dominating components for this model, tend to cancel in the secondorder contributions to the self-energy. The other extreme is model B (see eq.(㓙)), for which the second-order scalar term is even larger than the Hartree-Fock contribution to the scalar term. Since, however, the contributions of the second-order scalar and vector terms to the energy $p_{0}$ partly cancel each other, the total effect of the secondorder terms on $p_{0}$ is smaller than the Hartree-Fock term. Such a partial cancellation also occurs for the Lagrangian of model C (see eq.(5)), which is motivated from QCD. It should be kept in mind, however, that, in this case also, individual contributions are as large as 15 percent of the leading Hartree-Fock term. Substantial second-order effects are also observed in model D.

While the Hartree-Fock contribution to the self-energy yields a constant, the second-order contributions depend on the four-momentum $p$ of the quark under consideration. This implies that the quasiparticle energy, i.e., the self-consistent solution for $p_{0}, p_{0}^{Q P}(|\mathbf{p}|)$, obtained from eq.(31), is different from the quasiparticle energy for a free Dirac particle of mass $m^{*}$ or the quasiparticle energy resulting from a HartreeFock approximation, if the coupling constant is chosen to yield an effective mass $m^{*}$. To visualize the effects of the momentum-dependent self-energy on the quasiparticle energy $p_{0}^{Q P}(|\mathbf{p}|)$ we define an enhancement factor:

$$
\mathcal{E}(|\mathbf{p}|)=\frac{p_{0}^{Q P}(|\mathbf{p}|)}{\sqrt{|\mathbf{p}|^{2}+m^{* 2}}}
$$


This enhancement factor is displayed in the upper part of Fig. 6. As the coupling constants for all four Lagrangians were adjusted to yield a quasiparticle energy $p_{0}^{Q P}=$ $m^{*}$ for zero momentum, it is clear that all enhancement functions approach the value 1 in this limit. For larger momenta, however, the enhancement or better reduction factor deviates from this value indicating that the dependence of the self-energy on energy and momentum leads to quasiparticle energies which are quite different from those of a free particle.

It is worth noting that the larger fraction of this deviation is due to the vector component in the self-energy in models B and C. This can be seen by comparing the enhancement factors obtained in our complete calculation (upper part of the figure) to enhancement factors which are obtained if only the scalar part of the secondorder self energy is taken into account (see the lower part of the figure, where the corresponding enhancement factors are displayed, resulting from [8]). Also one may recall that the energy-dependence of the vector self-energy, which is an odd function of $p_{0}$, is generally larger than the corresponding scalar contribution, which is an even function of $p_{0}$ (see figure 4). From the enhancement factors displayed in Fig. 6, it is obvious that Lagrangians dominated by vector quark-quark interactions (models B and $\mathrm{C}$ ) tend to show much larger effects due to the second-order vector contributions than those which contain direct interaction terms of scalar and pseudoscalar form.

\section{Conclusions}

The self-energy of quarks is calculated for various models which are inspired by the Nambu-Jona-Lasinio (NJL) model, including terms up to second-order in the selfenergy of the quarks. The real and imaginary parts of scalar and vector components in the self-energy are discussed. It is demonstrated that the effects of the second-order terms are particularly large if the Lagrangian contains a strong vector quark-quark interaction. In such a model (Table 1, model B) individual contributions to the real part of the self-energy can even be larger than the Hartree-Fock contribution in the same model.

While the self-energy calculated in the Hartree-Fock approximation yields a constant, the contributions of second order display a characteristic dependence on the energy and momentum of the quark under consideration. The solution of the Dirac equations for quarks with such a self-energy leads to relations between the energy and

momentum of a quark which are different from that of a free Dirac particle with a constant effective mass. At small energies, it is the energy dependence of the vector component of the self-energy, in particular, which yields important modifications.

The second-order self-energies are directly related to the bare $q \bar{q}$ polarization propagators $\Pi_{\lambda}^{(0)}$ in the different excitation modes $\lambda$. If, in a next step, one replaces the bare propagators by the reducible polarization propagators as obtained in the RPA approximation, one expects a non-vanishing imaginary part in the self-energy at energies below the $2 q-1 \bar{q}$ threshold displayed, e.g., in Fig. 3. As the real and imaginary 
parts of the self-energy are related by dispersion relations (see eqs.(16) and (30)), this implies an even stronger energy dependence than that obtained in the approach presented here. Investigations in this direction are in progress.

This work has partly been supported by the Deutsche Forschungsgemeinschaft (DFG Fa 67/14-1); this support is gratefully acknowledged.

\section{References}

[1] Y. Nambu and G. Jona-Lasinio, Phys. Rev. 122345 (1961) ; 124246 (1961).

[2] S.P. Klevansky, Rev. Mod. Phys. 64, 649 (1992).

[3] R. Alkofer and H. Reinhardt, Z. Phys. A343 79 (1992).

[4] V. Bernard, U.-G. Meissner, and I. Zahed, Phys. Rev. D36, 819 (1987).

[5] T. Hatsuda and T. Kunihiro, Phys. Lett. B 185, 304 (1987).

[6] E.M. Henley and H. Müther, Nuc. Phys. A513 667 (1990).

[7] Nan-Wei Cao, C.M. Shakin, and Wei-Dong Sun, Phys. Rev. C46 2535 (1992).

[8] P.P. Domitrovich, D. Bückers, and H. Müther, Phys. Rev. C48 413 (1993).

[9] B.D. Serot and J.D. Walecka, Adv. Nucl. Phys. 16, 1 (1986).

[10] S.A. Chin, Ann. of Phys. 108, 301 (1977).

[11] R.T. Cahill and C.D. Roberts, Phys. Rev. D36 2419 (1985).

[12] M. Schaden, H. Reinhardt, P.A. Amundsen, and M.J. Lavelle, Nucl. Phys. B339, 595 (1990).

[13] H. Reinhardt, Phys. Lett. B248 365 (1990).

[14] D. Bückers and H. Müther, Nucl. Phys. A523 629 (1991). 
Table 1: Various contributions to the real part of the self-energy for quarks with momenta $\mathbf{p}=0$. The row labeled $\Sigma_{s}^{H F}$ shows the Hartree-Fock contribution, while the labels $\Sigma_{s}^{(2)}$ and $\Sigma_{0}^{(2)}$ refer to the scalar and vector terms, respectively, of the real part of the self-energy obtained in second-order. Results from the various Lagrangians A, $\mathrm{B}, \mathrm{C}$, and $\mathrm{D}$, discussed in section 2, are compared. For each Lagrangian the coupling constant has been chosen such that the self-consistent energy relation of eq.(31) yields $313 \mathrm{MeV}$. All entries are given in $\mathrm{MeV}$.

\begin{tabular}{lrrrr}
\hline \hline Term & Model A & Model B & Model C & Model D \\
& & & & \\
\hline$\Sigma_{s}^{H F}$ & 295.45 & 187.36 & 280.32 & 209.04 \\
$\Sigma_{s}^{(2)}$ & 10.67 & 203.60 & 44.97 & 87.25 \\
$\Sigma_{0}^{(2)}$ & -1.88 & 82.96 & 17.29 & -11.71 \\
& & & & \\
\hline \hline
\end{tabular}




\section{Figure Captions}

Figure 1: Diagrams representing the second-order contribution to the self-energy for a Fermion with momentum $p$. The labels for the momenta of the intermediate Fermion $(q)$ and the polarization and boson propagators $(p-q)$ are identical to those used in Section 3.

Figure 2: Imaginary parts of the scalar (left side) and vector (right side) self-energies for quarks with momenta $p$, assuming a scalar interaction (eq.(11)). For various values of $|\mathbf{p}|$, results are displayed as a function of $p_{0}$. The self-energy has been calculated assuming a Green's function for quarks characterized by a constant effective mass $m^{*}$ $=313 \mathrm{MeV}$, a cutoff parameter $\Lambda=653 \mathrm{MeV}$, and a coupling constant $\tilde{G}=1.618$ $\times 10^{-6} \mathrm{MeV}^{-2}$.

Figure 3: Imaginary parts of the scalar (left side) and vector (right side) self-energies for quarks obtained from scalar, pseudoscalar, and vector quark-quark interactions. Results are given for quarks with momentum $|\mathbf{p}|=0$ as a function of energy $p_{0}$. While the results for the vector component obtained from a vector interaction (line labeled with asterisks in the right part of the figure) can be read with the scale on the left-hand side, all other curves are related to the scale on the right-hand side of the figure. For further details see Figure 2.

Figure 4: Real parts of the scalar and vector self-energies for quarks with momentum $|\mathbf{p}|=0$, assuming various interactions. For further details, see the caption of Figure 3.

Figure 5: Real parts of the scalar and vector self-energies for quarks with momentum $p$, considering various interactions. Assuming $p_{0}=1 \mathrm{GeV}$, results are presented as a function of $|\mathbf{p}|$. For further details, see the caption of Figure 3.

Figure 6: Enhancement factor $\mathcal{E}$ for the quasiparticle energies as defined in eq.(32) as a function of the quark momentum $\mathbf{p}$ for the various Lagrangians discussed in Section 2. While the upper part of the figure displays the results obtained with inclusion of all second-order contributions, the results displayed in the lower part are obtained for the case when the vector component of the self-energy is ignored. 